\title{
Transmission of religion and attitudes
}

\author{
Lindon Eaves ${ }^{1}$, Brian D'Onofrio ${ }^{1}$ and Robert Russell ${ }^{2}$ \\ ${ }^{1}$ Virginia Institute for Psychiatric and Behavioral Genetics, Virginia Commonwealth University School of Medicine, \\ Richmond, Virginia \\ ${ }^{2}$ Center for Theology and the Natural Sciences, Berkeley, California, USA
}

The collection of papers in this special edition of Twin Research represents a small landmark in the study of behavioral genetics. The work published here is the fruit of an invited three-day workshop, supported by the John M Templeton Foundation, hosted by the Center for Theology and the Natural Sciences (Berkeley, November 13-15, 1998), which was specifically devoted to the task of placing varieties of religious experience and practice within a behavior-genetic context. Within the life and the behavioral sciences, the scientific study of religion has often been regarded as 'off limits'. From the perspective of a science that is still rallied by the stories of Galileo and Darwin, too much interest in religion might betoken an intrusive commitment to something other than scientific truth. From the standpoint of theology, science is often viewed as conveniently irrelevant at best and, at worst, destined only to erode still further the crumbling buttresses of religious tradition.

Both these views are inimical to science and the humanities. Religion and the values often associated with it represent towering accomplishments of biological and cultural evolution that are quintessentially 'human'. An understanding of 'the human' is impossible without an understanding of those qualities and institutions that, in many cultures, are still intimately connected with religious belief and practice. Anthropology and psychology that ignore religion deal neither with the anthropos in 'anthropology' nor the psyche in 'psychology' and 'psychiatry'.

It would be presumptuous to suggest that behavior genetics is going to revolutionize our understanding of religion and values. However, at the very least, the transmission of religion and values presents model systems that illustrate many of the subtleties and pitfalls in the study of biological and cultural inheritance. At the very best, we are addressing the material, evolutionary and historical foundation for humanity's fascination with the transcendent that, 30 years ago, the evolutionary geneticist Theodosius Dobzhansky named 'the Biology of Ultimate Concern'.

Correspondence: Dr Lindon Eaves, Virginia Institute for Psychiatric and Behavioral Genetics, Virginia Commonwealth University School of Medicine, Richmond, VA, USA
The papers in this volume are unified by their authors' common spirit of scientific fascination with the problem of relating biology to that which is commonly assumed to be purely 'cultural' and characteristically 'human'. The authors are also intrigued by the possibility that behavioral genetics can help unravel some of the complex mechanisms underlying the development of religion and values and perhaps yield some insight into their role in adaptation. The authors do not make any grandiose claims for the significance of their findings, nor do they pretend that they support one or other particular political, philosophical, theological or ethical perspective. Indeed, the contributors represent almost every shade and perspective current in western academia and are as divided about the implications of their work as any group of informed scholars.

The participants were selected because they were known to have unpublished data, mainly on large samples of twins, that might illuminate some issue in the scientific study or religion and values. Participants were given considerable freedom to develop whatever line of inquiry seemed most productive. The initial versions of the papers were circulated among participants, discussed intensively at the workshop and revised for publication. It was clear that the workshop model, where there was an intense focus on presenting and discussing models and data, was extremely rewarding and productive for the participants.

Existing published behavior-genetic studies have provided preliminary insight into the transmission of different religious constructs (reviewed in D'Onofrio et $\mathrm{al}^{1}$ ). Religious affiliation appears to be purely cultural, whereas religious attitudes and behavior, including church attendance, show varying degrees of genetic inheritance in addition to the influence of the shared environment and assortative mating. However, these findings are hindered in that many of the studies only included crude measures of religiousness. Likewise, little behavior-genetic research has addressed more complex issues, such as the relationship between personality/character and religiousness, religiousness as a multidimensional construct, differences in inheritance between ethnicities and cultures, developmental trends in religious expression or behavior, and the role religion 
plays in the etiology or perpetuation of clinical and behavior outcomes. The articles in this special issue begin to address these more intriguing methodological and research topics.

The paper by Eaves et al explores the role of genetic and environmental factors in personality and social attitudes in the Virginia30000 study. The findings of this large study of personality confirm what has been found in many other twin and family studies. Variation is partly genetic, but there are large effects of the within-family environment unique to individuals. However, the effects of the shared family environment and assortative mating are too small to be influential for the major dimensions of personality: psychoticism, extroversion and neuroticism. These results are in striking contrast to those for church attendance and social attitudes for which the role of assortment is extremely marked and for which some effects of the shared environment are implicated. Thus, the causes of variation in personality show a marked difference from those found with aspects of religion and social attitudes. Caution must be taken when considering these findings as consideration of more relevant personality dimensions may provide different results. In the second paper, Kirk et al show that a more explicitly 'religious' or 'spiritual' measure of personality, namely, self-transcendence, behaves quite unlike other religious and attitude measures and much more like other, more typical, personality measures in showing a moderate genetic component of variance and non-significant effects of the shared environment. In so far as self-transcendence measures 'spirituality' this finding supports the theoretical distinction between this characteristic and more organized religious behavior. Bouchard et al examine the transmission of intrinsic and extrinsic religiousness, an established distinction in the types of religious motivation within the field of the psychology of religion. The different pattern of inheritance in these two phenomena provide more evidence that religiousness is multidimensional. Likewise, these two religious measures did not correl ate highly with other personality constructs, further supporting the separation of the religion and personality domains.

In order to delineate cultural differences in church attendance, Kirk et al report the joint analysis of two large sets of data on church attendance comprising a total of nearly 50000 twins and their relatives from more than 11000 families in the USA and Australia. Some of the findings are qualitatively similar in the two populations; for example, both studies arrive at the same surprising conclusion that at least some of the variation in church attendance is partly genetic, and that the role of assortative mating is very marked in both populations. The populations differ, however, in the relative contribution of the environment provided by mothers and fathers. The paper by Viken et al examines familial effects on religiousness in 2393 pairs of 16 -year-old Finnish twins. Overall, they show very large effects of the shared environment and very small genetic effects, but note that there are regional differences in the relative contributions of genetic and within-family environmental differences. These findings underscore the fact that there is no reason to suppose that genetic and environmental parameters are universal constants but will vary as a function of biological and cultural history. Crudely, church attendance is a function of individual priorities in life which may well be shaped in part by innate differences in temperamental characteristics.

The intriguing paper by Boomsma et al addresses the influence of religious affiliation in a culture where there are very marked differences in life style between those who identify with the Calvinist tradition and those who do not. Familial differences in religious affiliation are entirely due to the effects of the shared environment. Of special significance, however, is their demonstration that a religious upbringing apparently reduces the expression of genetic differences on measures of disinhibition. That is, there is evidence for an interaction between the shared environment reflected in a religious upbringing and the expression of genetic differences on personality. It is tempting to see this as an example of what Cattell once called 'cultural coercion to the biosocial norm' within the religious tradition. That is, the apparent genotype $\times$ environment $(G \times E)$ interaction could result from the fact that religious families tend to make a more significant effort to modify the behavior of those whom the random variations of genetic segregation predispose to more extreme behavioral patterns (negative genotype-environment correlation). If this finding is replicated, it suggests that religion may offer a model system for exploring the impact of $G \times E$ interaction and $G-E$ correlation in behavioral development. A further facet of the impact of cultural differences on the expression of genetic factors is demonstrated in a later paper by Heath et al. Among other things, they show that the heritability of religious involvement is greater in adolescent African American girls than in Americans of European and other ancestry.

It is becoming clear that various aspects of religion are an often neglected protective factor for several negative behavioral outcomes. McCullough and Larson review the growing literature on religion and depression. Although they note that the standards in such research still leave much to be desired, religious affiliation, high levels of religious involvement, high extrinsic religious motivation and religious salience all confer modest protection against depression. The authors also provide a critical look at the 
methodological and quantitative issues involved in studying the relationship between religiousness and behavior.

Four papers in this collection begin to tease apart some of the subtleties and mechanisms underlying this relationship. Kendler et al show how different aspects of religion - personal devotion, institutional conservatism and personal conservatism - influence the depressogenic effects of different types of stressful life events. They note that high levels of personal devotion and institutional conservatism protect against the effects of death and personal illness, whereas high levels of personal conservatism are associated with increased sensitivity to relationship problems. Heath et al demonstrate a marked protective effect of religious involvement and values against adolescent alcohol use. Furthermore, their data suggest that the greater religious involvement of African American adolescent girls partly explains the lower use of alcohol in this population. D'Onofrio et al explore the nature of adolescent religiousness and some aspects of the relationship between religiousness and substance use in this age group. They intimate that specific religious beli efs about the sinfulness of drug use and levels of peer religiousness mediate the impact of belief in God and religious practices on substance use. With data from the Virginia30000, Maes et al specify the genetic and environmental correlations between church attendance and alcohol use with their bivariate comprehensive model. Gender differences were found since genetic factors account for the association between these variables in males, whereas the relationship is due to shared environmental factors and genotype-environment covariance in females. These intriguing findings only begin to tease apart the association between religiousness and different behaviors.

The papers in this volume are still too scattered to provide anything greater than hints of the overall landscape but they al ready suggest a rich territory for further inquiry. As a subject of inquiry, religiousness is a complex and multidimensional phenotype. We see in religious affiliation the elements of a model system in which transmission is almost exclusively cultural but whose effects may moderate the expression of genetic differences on personality. In religious attitudes and practice, we observe patterns of behavior for which the effects of genes and environment are dependent on age, gender, ethnicity, nationality and context. What is astonishing to most of us is that even in the most 'cultural' of variables, there are circumstances in which the effects of genes cannot be ignored. In the studies of religion and outcomes such as substance use and psychopathology, we are beginning to see clues that take us beyond a mere catalog of associations into models for the role of religion in human development and adaptation.

We suspect that there are other investigators who have measured variables related to religion in other contexts who, like us, have paid scant attention to their significance as model systems for the interaction of genetic and social factors in human variation or to their potential role as variables mediating or modulating genetic risk to clinically important outcomes. It is our hope that the papers in this collection will motivate others to develop still more subtle models for the roles of genes and environment in human adaptation.

\section{References}

1 D'Onofrio BM, Eaves LJ Murrelle L, Maes HH, Spilka B. Understanding biological and social influences on religious affiliation, attitudes, and behavior: A behavior-genetic perspective. J Personality (in press). 